VIII ${ }^{\text {èmes }}$ Journées Nationales Génie Civil - Génie Côtier, Compiègne, 7-9 septembre 2004

\title{
Inter-comparaisons de mesures de vagues au large du Cap Ferret
}

\author{
Hélène Dupuis(a), Gérard Goasguen(b), Loïc Michel(c), \\ Bertrand Michard(d), Patrice Bretel(e)
}

\author{
(a)Université Bordeaux 1-UMR 5805, avenue des facultés, 33405 Talence cedex \\ (b)CETMEF - Technopole Brest-Iroise, BP 5 - 29280 Plouzané \\ (c)R.D. Instruments Europe, Les Nertières, 06610 La Gaude \\ (d)CETMEF - Technopole Brest-Iroise, BP 5 - 29280 Plouzané \\ (e)Université Bordeaux 1-UMR 5805, avenue des facultés, 33405 Talence cedex
}

\section{Résumé}

Une inter-comparaison entre trois houlographes directionnels situés près de la côte Atlantique française, par 56 mètres de fond, a été effectuée sur des données enregistrées en 2001 et 2002. Deux des houlographes sont des bouées : une Datawell Waverider (MK II) et une Triaxys de chez Axys technologies. Le troisième, posé sur le fond, est un ADCP WHsentinel, de RD Instruments, fonctionnant à $300 \mathrm{KHz}$.

Les comparaisons ont été réalisés en prenant pour référence la bouée Datawell Waverider directionnelle puisque ce système validé est le plus ancien et le mieux connu.

Les corrélations pour les hauteurs significatives, périodes moyennes et paramètres de pic (période, direction) sont respectivement très bonne, bonne et pauvre. L'accord est toujours meilleur entre les deux bouées de surface qu'entre la Datawell et l'ADCP posé sur le fond.

Pour l'ADCP, les hauteurs significatives obtenues par la méthode de détection de surface ou celle des vitesses orbitales sont similaires. La période moyenne semble sous-estimée d'une seconde par tandis que le biais sur la période de pic est seulement de 0,1 seconde. L'ADCP a l'avantage de fournir en plus des paramètres de vagues, les profils de courants dans la colonne d'eau.

Les différences sur les paramètres de pic sont principalement constatées sur les basses fréquences $(\mathrm{f}<0.1 \mathrm{~Hz})$ pour la bouée Triaxys (beaucoup de périodes pics supérieures à 20s) et sur les hautes fréquences pour l'ADCP (par exemple les biais sur les périodes moyennes sont plus important que sur les périodes pics).

\section{$\underline{\text { Abstract }}$}

Three different directional wave sensors were compared in 2001and 2002 at a coastal location in $56 \mathrm{~m}$ water depth off the Atlantic French coast. Both of them are directional buoys: a Datawell wave rider (MK II) and a Triaxys (Axys technologies), while the third one is a bottom mounted ADCP (WH-sentinel autonomous, from RD Instruments, working at 300KHZ).

The comparisons are based on the Datawell wave rider as a reference since it is the oldest and best known and validated system.

Correlations for significant wave heights, mean periods and peak parameters (period and directions) are respectively very good, good and poor. The agreement is always better between the two surface buoys than between the Datawell buoy and the bottom mounted ADCP. However, the ADCP also provides current profiles in the water column.

For the ADCP results are rather similar for significant wave heights as obtained by surface tracking or with orbital velocities. For the wave period the mean wave period seems to be underestimated by 1 second while the bias for the peak period is only from 0.1 second. 
Discrepancies for wave parameters seem to be mostly located at low frequencies $(\mathrm{f}<0.1$ $\mathrm{Hz}$ ) for the Triaxys buoy and at high frequencies for the ADCP.

\section{Introduction}

L'Université de Bordeaux 1 (UMR 5805), en collaboration avec le Centre d'Etudes Techniques Maritimes et Fluviales (CETMEF), réalise depuis août 2001 des mesures d'états de mer directionnels, par $56 \mathrm{~m}$ de fond, à 10 milles au large du Cap-ferret. Ce dispositif opérationnel, utilisé également pour la recherche, a conduit à une mise à disposition des données en temps réel sur le site http://www.cetmef.equipement.gouv.fr/donnees/candhis/. Le déploiement de ces mesures en continu de la houle directionnelle est nécessaire pour décrire les états de mer qui forcent la dynamique littorale. Les précédentes mesures utilisées pour quantifier la statistique des états de mer (Butel et al, 2002) se basaient sur des mesures non directionnelles, complétées par les sorties de modèles numériques pour le paramètre de direction de vagues qui joue un rôle important, en particulier pour la dérive littorale.

L'acquisition d'un matériel relativement récent (la bouée directionnelle Triaxys de chez Axys technologies) peu utilisé en recherche, nous a incité à mettre en œuvre une intercomparaison de près de six mois avec le standard de fait pour les bouées de surface en eau de profondeur intermédiaire: la bouée Datawell Waverider directionnelle (MK II). Les instruments ont été comparés dans une zone pouvant être considérée comme homogène spatialement du point de vue du champ de vagues puisqu'ils étaient déployés à une distance maximale de $200 \mathrm{~m}$ les uns des autres (voir dispositif expérimental sur le site http://www.epoc.u-bordeaux.fr/, équipe METHYS, «bouée »).

La mise en œuvre sur le site, du 11 avril au 12 juillet 2002, d'un ADCP-WH-sentinel autonome, de RD Instruments, fonctionnant à 300KHZ, utilisé pour la mesure des états de mer a permis d'étendre cette inter-comparaison.

Les inter-comparaisons ont été réalisées sur des échantillons d'états de mer assez représentatifs de la zone avec des maxima de hauteurs significatives de l'ordre de $6 \mathrm{~m}$ par les houlographes de surface, pour la période de six mois, et de $5 \mathrm{~m}$ pour l'ADCP, pour une période de 3 mois.

Dans cet article nous présenterons successivement les inter-comparaisons des deux houlographes de surface et de l'ADCP avec la bouée Datawell sur les paramètres hauteurs significative, période moyenne et directions calculés dans le domaine spectral.

\section{Inter-comparaison de la bouée TRIAXYS avec la bouée Datawell Waverider directionnelle}

Les deux bouées de mesure d'états de mer directionnels, du type à trois composantes de déplacement, sont équipées de modules de calcul embarqués et transmettent à la station réceptrice un certain nombre de résultats, obtenus soit dans le domaine temporel, soit dans le domaine spectral.

Les paramètres spectraux produits par les deux systèmes ne sont pas toujours calculés de la même façon. Pour élargir la comparaison, nous avons donc choisi de recalculer tous les paramètres, à partir des spectres $1 \mathrm{D}$ (disponibles pour les deux bouées) et 2D (disponibles pour TRIAXYS, tandis que Datawell propose les deux premières harmoniques angulaires pour chaque fréquence).

Les gammes de fréquences choisies par les deux constructeurs ne sont pas identiques. Nous avons donc utilisé, à la fois, les gammes de fréquences proposées par les constructeurs, mais également appliqué la gamme de fréquences Datawell pour recalculer les paramètres Triaxys de manière à offrir une comparaison plus cohérente.

La période de comparaison s'étend du 28 août 2001 au 29 janvier 2002, elle est représentative des conditions hivernales et nous avons rencontré des hauteurs significatives 
supérieures à $6 \mathrm{~m}$. La durée des enregistrements est de 30 minutes toutes les heures, avec un décalage de 12 minutes entre les deux systèmes.

\subsection{Les paramètres du spectre 1D : hauteurs et périodes}

\subsubsection{Définition des paramètres calculés pour chacune des bouées.}

A partir du spectre 1D, nous avons recalculé les paramètres décrits dans la table 1, voir Tucker (1991) pour plus de détails.

Table 1: Paramètres du spectre $1 D$

\begin{tabular}{|c|c|c|c|}
\hline Symbole & Titre & Description & $\begin{array}{c}\text { Moment de } \\
\text { définition }\end{array}$ \\
\hline $\mathrm{H}_{\mathrm{m} 0}$ ou $\mathrm{Hs}\left(\mathrm{m}_{0}\right)$ & Hauteur significative & $\begin{array}{l}\text { Calcul de Hs par analyse } \\
\text { spectrale }\end{array}$ & $4\left(m_{0}\right)^{1 / 2}$ \\
\hline $\mathrm{T}_{01}$ ou $\mathrm{T}_{\text {moy }}$ & Période moyenne. & $\begin{array}{l}\text { Inverse de la fréquence moyenne } \\
\text { du spectre. }\end{array}$ & $\mathrm{m}_{\mathrm{o}} / \mathrm{m}_{1}$ \\
\hline $\mathrm{T}_{\mathrm{p}}$ & $\begin{array}{l}\text { Période de pic à partir de la } \\
\text { valeur de fp. }\end{array}$ & $\begin{array}{l}\text { Inverse de la fréquence fp où } S(f) \\
\text { atteint sa valeur maximale. }\end{array}$ & \\
\hline
\end{tabular}

avec $\mathrm{m}_{\mathrm{n}}$ moment d'ordre $\mathrm{n}$ du spectre défini par :

$$
\mathrm{m}_{\mathrm{n}}=\int_{0}^{\infty} \mathrm{f}^{\mathrm{n}} \mathrm{S}(\mathrm{f}) \mathrm{df}
$$

\subsubsection{Résultats des comparaisons}

Comme l'indique la table 2, la corrélation est très bonne pour les hauteurs significatives $\mathrm{H}_{\mathrm{m} 0}$. L'erreur relative moyenne se situe vers les $7 \%$, tandis que l'erreur absolue moyenne (erreur RMS) est de l'ordre de $13 \mathrm{~cm}$. Les biais sont très faibles (inférieurs à $2 \mathrm{~cm}$ ) et changent de signe selon la gamme de fréquence utilisée. Le fait d'utiliser la même gamme augmente très légèrement la corrélation des deux paramètres, mais augmente le biais.

On constate deux tendances pour les périodes :

On obtient une bonne corrélation pour les périodes moyennes $\mathrm{T}_{01}$. L'erreur relative moyenne se situe vers les 5\%, tandis que l'erreur absolue moyenne est de l'ordre de 0,4 s. Les biais augmentent jusqu'à 0,2 secondes, lorsque l'on utilise la même gamme de fréquence pour les deux instruments.

La corrélation est beaucoup moins bonne pour la période du pic $\mathrm{T}_{\mathrm{P}}$. La loi de régression s'éloigne largement de l'unité (pente, "a", de l'ordre $0.4 \mathrm{~T}_{\mathrm{P}}$ ).

En effet, la bouée Triaxys donne parfois des valeurs aberrantes de la période de pic (voir la Figure 1c). On obtient des valeurs supérieures à 20 secondes dans la gamme des basses fréquences. Ces valeurs aberrantes sont obtenues dans le cas de mers très peu énergétiques où on mesure vraisemblablement les rappels du mouillage dont l'origine est liée à un mauvais filtrage des basses fréquences. Le fait de supprimer ces valeurs permet d'obtenir une loi de régression proche de l'identité. Cependant, la dispersion sur ce paramètre reste importante comme l'indique le nuage de point principal de la figure $1 \mathrm{c}$, du fait du faible degré de liberté sur ce paramètre entraînant une plus forte incertitude sur la fréquence pic. Ce paramètre est pourtant couramment utilisé car plus représentatif pour les états de mer complexes (superposition de plusieurs trains de houle) observé sur les sites océaniques ouverts. 
Table 2 : comparaison des paramètres spectraux recalculés sur les spectres $1 D$ des deux bouées sur leurs gammes de fréquence totales (non exactement similaires) pour la première ligne de chaque paramètres ou bien, pour la deuxième ligne, sur la gamme de fréquence de la bouée Datawell dans les deux cas.

\begin{tabular}{|l|c|c|c|c|c|c|c|c|c|c|}
\hline & $\mathbf{a}$ & $\mathbf{b}$ & $\mathbf{S a}$ & $\mathbf{S b}$ & $\mathbf{R}^{2}$ & $\mathbf{E r m}$ & $\begin{array}{c}\text { scatter } \\
\text { Index(\%) }\end{array}$ & $\begin{array}{c}\text { ErRm } \\
(\boldsymbol{\%})\end{array}$ & $\begin{array}{c}\text { ErR } \\
(\boldsymbol{\%})\end{array}$ & ERMS \\
\hline $\mathbf{H}_{\mathbf{m} \mathbf{0}}$ & 1.008 & -0.0100 & 0.0022 & 0.0044 & 98.4 & 0.004 & 7.45 & -0.24 & 6.78 & 0.128 \\
\hline & 0.996 & -0.0087 & 0.0022 & 0.0044 & 98.4 & -0.015 & 7.29 & -1.21 & 6.54 & 0.127 \\
\hline $\mathbf{T}_{\mathbf{0 1}}$ & 1.021 & -0.2620 & 0.0029 & 0.0224 & 97.5 & -0.102 & 4.93 & -1.85 & 5.43 & 0.382 \\
\hline & 1.031 & -0.4327 & 0.0030 & 0.0236 & 97.3 & -0.201 & 5.08 & -3.29 & 5.71 & 0.433 \\
\hline $\mathbf{T}_{\mathbf{p}}$ & 0.414 & 6.5704 & 0.0117 & 0.1445 & 27.7 & -0.288 & 30.03 & 1.62 & 24.57 & 3.525 \\
\hline & 0.399 & 6.7176 & 0.0118 & 0.1460 & 26.1 & -0.345 & 30.47 & 1.15 & 24.24 & 3.599 \\
\hline
\end{tabular}

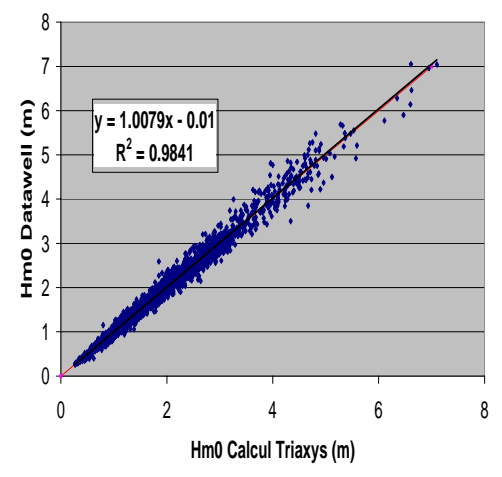

(a) Hauteur significative

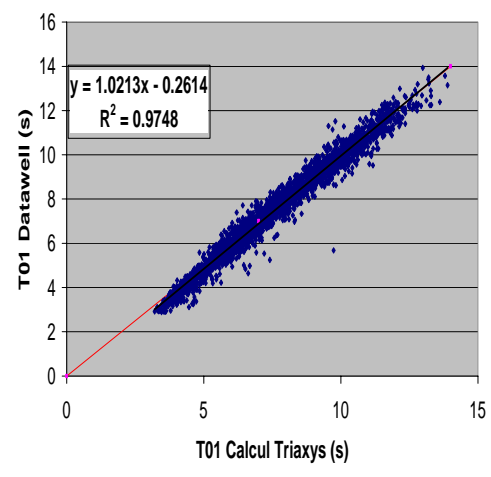

(b) Période moyenne

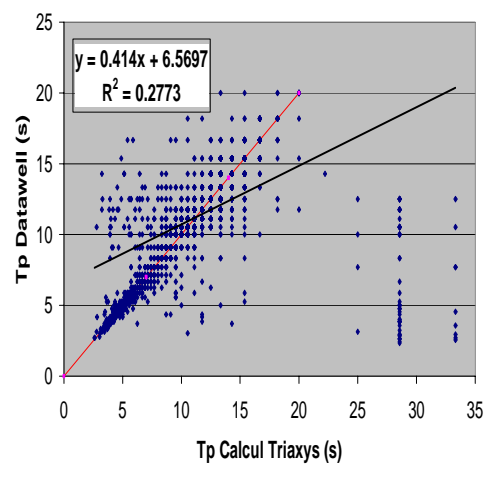

(c) Période de pic

Figure 1 : Diagrammes de dispersion des paramètres hauteur significative, période moyenne et période au pic. Données de la bouée Triaxys en abscisse, et Datawell en ordonnées. Sur chaque graphique, on indique la droite identité et la droite de régression dont les paramètres sont décrits dans la table ci dessus.

\subsection{Le paramètre direction issue du spectre 2D}

\subsubsection{Définition des paramètres calculés pour chacune des bouées.}

Le spectre directionnel peut être défini par

$S(f, \theta)=S(f) \cdot D(f, \theta)$ où $\int_{-\pi}^{+\pi} D(f, \theta) d \theta=1$

avec $\quad \mathrm{S}(\mathrm{f})$ Densité spectrale

$D(f, \theta)$ fonction de répartition angulaire 
On définit la direction moyenne $\theta_{\mathrm{m}}$, pour chaque fréquence, en partant de la première harmonique de la décomposition en série de Fourier de la fonction de répartition angulaire par:

$$
\theta_{m}(f)=a \tan \left(b_{1} / a_{1}\right)
$$

La direction au pic est égale à la direction moyenne $\theta_{\mathrm{m}}$ de la bande de fréquence du pic du spectre d'énergie.

On obtient la direction moyenne globale en pondérant la somme des directions moyennes par l'énergie, on a :

$$
\theta_{m}=\frac{\int_{0}^{\infty} S(f) \theta m(f) d f}{\int_{0}^{\infty} S(f) d f}
$$

La bouée Datawell fournit la direction au pic et les coefficients de Fourier de la première harmonique de la fonction de répartition angulaire. Le système Triaxys donne la direction moyenne globale et le spectre 2D.

Pour pouvoir comparer les résultats, nous avons donc été amené à calculer, pour la Datawell, la direction globale moyenne à partir des coefficients de Fourier produits, .pour la Triaxys, à calculer la direction au pic à partir des coefficients de Fourier déduits du spectre $2 \mathrm{D}$.

\subsubsection{Comparaison des paramètres directionnels.}

Les paramètres statistiques décrivant les régressions linéaires des paramètres directionnels sont décrits dans la table ci-dessous et la figure montre les diagrammes de dispersions pour les directions au pic ou en moyenne. On a retiré les erreurs relatives qui n'ont pas trop de sens avec le modulo $2 \pi$.

On remarque encore une fois que la dispersion est très importante au pic spectral avec des erreurs RMS jusqu'à $22^{\circ}$ pour la direction au pic $^{\circ}$, en partie lié au fait que, dans les cas de mers complexes avec des trains dont l'énergie est comparable, la fréquence pic identifiée par les différents systèmes peut correspondre à des trains de vagues différents.

Pour la direction moyenne globale, la corrélation est bien meilleure, ainsi que la dispersion, avec une erreur RMS de $11^{\circ}$. Le biais est de $6^{\circ}$.

Table 3 : comparaison des paramètres directions recalculés pour les deux bouées

\begin{tabular}{|c|c|c|c|c|c|c|c|}
\hline & $\mathbf{a}$ & $\mathbf{b}$ & $\mathbf{S a}$ & $\mathbf{S b}$ & $\mathbf{R}^{2}$ & $\mathbf{E r m}$ & ERMS \\
\hline$\theta_{\mathbf{p i c}}$ & 1.155 & -52.806 & 0.0092 & 2.672 & 89.7 & -8.355 & 22.454 \\
\hline$\theta_{\mathbf{m}}$ & 0.942 & 10.9622 & 0.0055 & 1.653 & 94.2 & -6.477 & 11.034 \\
\hline
\end{tabular}



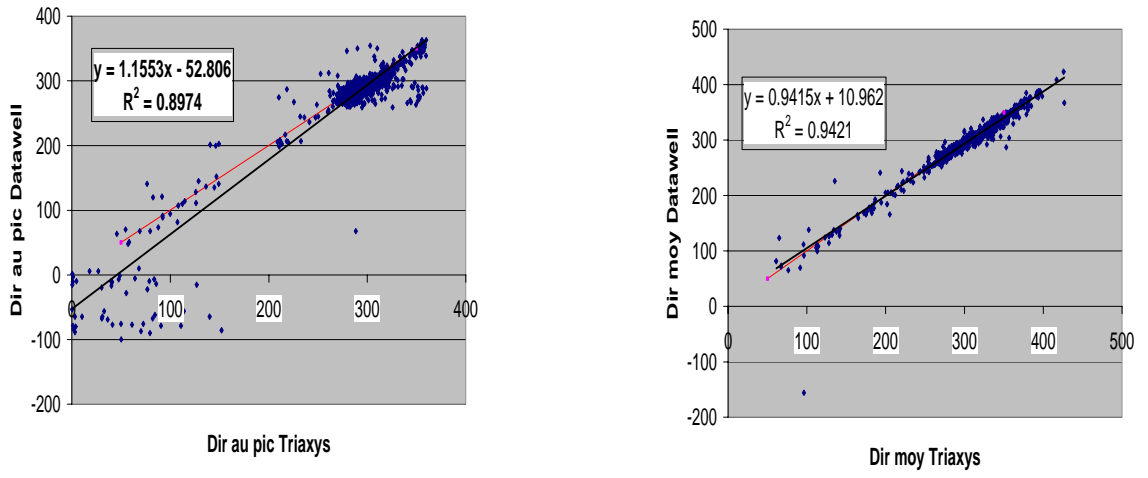

Figure 2 : diagrammes de dispersion des directions. Données de la bouée Triaxys en abscisse, et Datawell en ordonnées. Sur chaque graphique, on indique la droite identité et la droite de régression dont les paramètres sont décrits dans la table ci dessus.

\section{Inter-comparaison de l'ADCP RDI avec la bouée Datawell Waverider directionnelle}

\subsection{Méthodes utilisées par l'ADCP}

Le profileur de courant à effet Doppler (ADCP), de RD Instrumentation, utilise trois méthodes différentes pour estimer les états de mer : la mesure des vitesses orbitales induites par la houle en différents points de la colonne d'eau, la détection de la surface libre par le temps de propagation d'un signal acoustique, et la mesure de la pression. La corrélation de ces trois méthodes avec les mesures Datawell est utilisée pour valider le résultat. Ces méthodes utilisent le potentiel des profileurs acoustiques incluant un capteur de pression. Une estimation du spectre unidirectionnel des vagues est fournie par le capteur de pression mais qui, du fait de la profondeur d'eau (-56 m) et de l'amortissement des fluctuations de pression avec la profondeur en condition non hydrostatiques, n'est sensible qu'aux fluctuations de surface de grandes longueur d'ondes. Les deux autres méthodes, respectivement appelées par la suite « vitesses » et « surface », sont plus appropriées à ces profondeurs et se basent sur la théorie linéaire de 5 mesures de vitesses orbitales dans chacun des 4 faisceaux ou des 4 échos de surface permettant de déterminer l'élévation de la surface en 4 points. La direction des vagues n'est pas établie à partir de la méthode de vitesses.

Les signaux sont traités dans le domaine spectral. L'inter-comparaison est donc limitée aux paramètres suivants : Hauteur significative $(\mathrm{Hm} 0)$, période moyenne T01, période de pic et direction au pic.

La période de comparaison s'étend du 30 avril 2002 au 11 juin 2002.Nous avons rencontré des hauteurs significatives supérieures à $5 \mathrm{~m}$. La durée des enregistrements est respectivement de trente minutes et de vingt deux minutes toutes les heures pour la Datawell et pour l'ADCP. Le début des enregistrements est décalé de 12 minutes entre les deux systèmes.

\subsection{Résultats des comparaisons}

La corrélation est bonne pour les hauteurs significatives $\mathrm{H}_{\mathrm{m} 0}$ calculés par la méthode de détection de surface ou celle des vitesses orbitales. Les erreurs relatives moyennes sont de 
l'ordre de $11 \%$, tandis que l'erreur absolue moyenne est de l'ordre de $25 \mathrm{~cm}$. Les biais sont de 10 à $15 \mathrm{~cm}$ et changent de signe selon la méthode utilisée. Ces résultats sont assez comparables à ceux obtenus par un profileur Doppler concurrent (Pedersen et al., 2002). Les hauteurs significatives obtenues par la mesure de pression sont logiquement mois bonnes, quoique favorisées par un site océanique soumis à des houles généralement longues (Butel et al., 2002). Elles sont statistiquement biaisées avec une sous-estimation moyenne de l'ordre de $44 \mathrm{~cm}$ du fait de la sous-estimation systématique des petites longueurs d'onde.

On obtient une moins bonne corrélation pour la période moyenne $\mathrm{T}_{01}$ obtenue par la méthode de «surface » qui donne les meilleurs résultats. L'erreur relative moyenne se situe vers les $10 \%$, tandis que l'erreur absolue moyenne est supérieure à la seconde. Cette dispersion de l'ordre d'un facteur 2 par rapport à la comparaison des flotteurs de surface. Le principal problème de l'estimation de la période moyenne des vagues par ADCP réside dans le biais qui est de l'ordre de la seconde (sous estimation). La méthode de vitesses ne donne pas de meilleurs résultats, les biais sont opposés mais la dispersion est encore un peu plus importante.

On remarque encore que les résultats sont beaucoup moins bon au pic spectral du point de vue de la dispersion. Par contre aucun biais significatif n'est identifié sur la période au pic (0.1 seconde), ce qui laisse à penser, en accord avec la sur-estimation des hauteurs significatives par la méthodes des surfaces $(\mathrm{de} 15 \mathrm{~cm})$ que cette technique tend à légèrement sur-estimer l'énergie des hautes fréquences. Ce point devra donc être approfondi dans la suite de l'étude par une comparaison des spectres.

Table 4 : comparaison des paramètres spectraux pour la Datawell et l'ADCP

\begin{tabular}{|l|c|c|c|c|c|c|c|c|c|c|}
\hline & $\mathbf{a}$ & $\mathbf{b}$ & $\mathbf{S a}$ & $\mathbf{S b}$ & $\mathbf{R}^{\mathbf{2}}$ & $\mathbf{E r m}$ & $\begin{array}{c}\text { scatter } \\
\text { Index(\%) }\end{array}$ & $\begin{array}{c}\text { ErRm } \\
(\mathbf{\%})\end{array}$ & $\begin{array}{c}\text { s(ErR) } \\
(\mathbf{\%})\end{array}$ & ERMS \\
\hline $\begin{array}{l}\mathbf{H}_{\mathbf{m} \mathbf{0}} \\
\text { Surface }\end{array}$ & 1.008 & -0.1677 & 0.0084 & 0.0198 & 94.2 & -0.150 & 10.48 & -8.33 & 14.08 & 0.274 \\
\hline $\begin{array}{l}\mathbf{H}_{\mathbf{m} 0} \\
\text { Vitesses }\end{array}$ & 0.944 & 0.1953 & 0.0072 & 0.0153 & 95.3 & 0.091 & 11.82 & 8.99 & 16.79 & 0.239 \\
\hline $\begin{array}{l}\mathbf{H}_{\mathbf{m} 0} \\
\text { Pression }\end{array}$ & 0.938 & 0.5336 & 0.0117 & 0.0211 & 86.7 & 0.439 & 23.20 & 46.23 & 49.12 & 0.565 \\
\hline $\begin{array}{l}\mathbf{T}_{\mathbf{0 1}} \\
\text { Surface }\end{array}$ & 0.934 & 1.5285 & 0.0134 & 0.0900 & 84.4 & 1.098 & 9.91 & 18.30 & 12.52 & 1.274 \\
\hline $\begin{array}{l}\mathbf{T}_{\mathbf{0 1}} \\
\text { Vitesses }\end{array}$ & 1.066 & -1.82 & 0.0224 & 0.2009 & 72.5 & -1.234 & 9.53 & -14.31 & 9.86 & 1.497 \\
\hline $\begin{array}{l}\mathbf{T}_{\mathbf{p}} \\
\text { Surface }\end{array}$ & 0.635 & 3.7789 & 0.0190 & 0.2072 & 55.6 & -0.094 & 16.94 & 2.56 & 26.78 & 1.797 \\
\hline $\begin{array}{l}\mathbf{T}_{\mathbf{p}} \\
\text { Vitesses }\end{array}$ & 0.667 & 3.3035 & 0.0204 & 0.2250 & 55.3 & -0.267 & 15.79 & -0.52 & 19.66 & 1.714 \\
\hline $\begin{array}{l}\mathbf{D}_{\mathbf{p}} \\
\text { surface }\end{array}$ & 0.057 & 272.44 & 0.0155 & 4.5034 & 1.6 & -0.019 & 11.61 & 8.80 & 168.09 & 33.528 \\
\hline
\end{tabular}



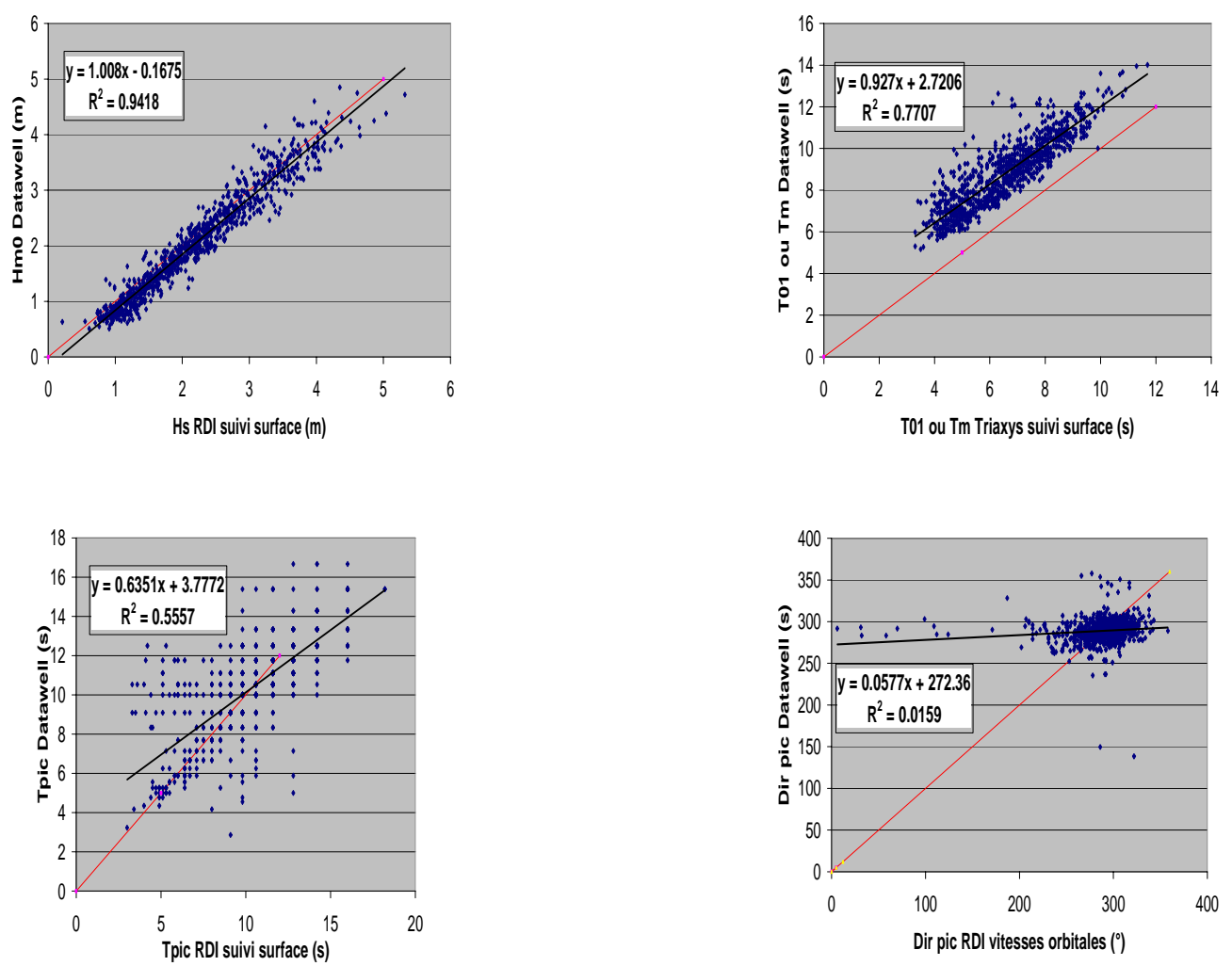

Figure 3 : Diagrammes de dispersion des paramètres hauteur significative, période moyenne, période au pic et direction au pic. Données de l'ADCP en abscisse avec la méthode de "surface », et Datawell en ordonnées. Sur chaque graphique on indique la droite identité et la droite de régression dont les paramètres sont décrits dans la table ci dessus

Les résultats sur la direction au pic ne sont pas très bons (à comparer à ceux de Gordon. et Lohrmann, 2001) et devront faire l'objet d'une analyse ultérieure.

\section{Conclusion}

Les campagnes d'instrumentation réalisées au large du Cap Ferret on permis d'effectuer des inter-comparaisons respectivement entre les bouées de mesure directionnelle d'états de mer Datawell et Triaxys, et entre Datawell et l'ADCP de RDI fonctionnant à $300 \mathrm{KHz}$.

La corrélation est très bonne à bonne pour les hauteurs significatives.

On obtient également une bonne corrélation pour les périodes moyennes entre Datawell et Triaxys. Les résultats sont de moindre qualité entre Datawell et l'ADCP.

La corrélation est beaucoup moins bonne pour la période de pic Tp.

Pour la direction au pic, on remarque encore que la dispersion est très importante. La corrélation est bien meilleure pour la direction moyenne globale.

La suite de cette étude devra comporter une comparaison systématique des spectres (Krogstad et al., 1999) et envisager d'étendre les comparaisons à une plus large gamme de paramètres, incluant par exemple Tp5, significative des plus hautes énergie du spectres mais moins bruitée que la période pic et la direction moyenne pour l'ADCP. Elle devrait nous 
permettre d'améliorer nos connaissances sur les différences constatées dans le domaine des hautes et basses fréquences (respectivement pour l'ADCP et la Triaxys) et au pic du spectre. Elle sera également étendue à l'étude de l'étalement directionnel.

\section{Références}

1.Association Internationale de Recherches Hydrauliques - Paramètres des états de mer

Supplément au bulletin $\mathrm{n}^{\circ} 52-1986$

2.Butel R., Dupuis H. et Bonneton P., Spatial variability of wave conditions at French Atlantic coast using in-situ data, J. of Costal Research, SI 36 , pp96-108, 2002 (ICS 2002).

3.Gordon, L. et Lohrmann A., Near-Shore Doppler Curent Meter Wave Spectra, ASCE Waves 2001 Conference, September 2001, 12pp.

4.Krogstad H. E., J. Wolf, S. P. Thompson et L. R. Wyatt, Methods for intercomparison of wave measurements, Cosatal Engineering 37, pp 235-257, 1999.

5.Pedersen, T., Nylund, S. et Dolle, A., Wave Height Measurements Using Acoustic Surface Tracking,MTS/IEEE Ocean '02 conference, Mississippi, October 2002, p 1234-1241.

6.Tucker, M.J. 1991. Waves in ocean engineering: measurement, analysis, interpretation.Chichester, England: Ellis Horwood Ltd, 430p.

\section{ANNEXE : Définition des différentes erreurs calculées}

Les erreurs définies ci après permettent de comparer les paramètres

$\mathbf{a}=$ Coefficient de corrélation

Obtenue a partir de l'équation de la courbe de tendance

$\mathbf{S a}=$ Incertitude de $\mathbf{a}$

$\mathbf{b}=$ Constante de corrélation

Obtenue de la même manière que a

$\mathbf{S b}=$ Incertitude de $\mathbf{b}$

$\mathbf{R}^{2}=$ Coefficient de détermination

Erm $($ Erreur moyenne $)=$ moyenne $[(\mathbf{y}-\mathbf{x})]$

Scatter index $($ indice de dispersement $)=$ écart type $[(\mathbf{y}-\mathbf{x}) / \operatorname{avg}(\mathbf{x})] * \mathbf{1 0 0}$

ErRm $($ Erreurs relatives $)=$ moyenne $((\mathbf{y}-\mathbf{x}) / \mathbf{x}) * \mathbf{1 0 0}$

ErR $($ Erreurs réelles $=$ écart type $)=$ écart type $[(\mathbf{y}-\mathbf{x}) / \mathbf{x}] * 100$

ERMS $($ Erreurs RMS $)=\operatorname{sqrt}\left(\right.$ moyenne $\left.\left[(\mathbf{y}-\mathbf{x})^{2}\right]\right)$ 